Vázquez Cano, E., Sevillano García, M. L. y Fombona Cadavieco, J. (2016). Análisis del uso educativo y social de Tos dispositivos digitales en el contexto universitario panhispánico. Revista de Investigación Educativa, 34(2), 453-469. DOI: http://dx.doi.org/10.6018/rie.34.2.224691

\title{
Análisis del uso educativo y social de los dispositivos digitales en el contexto universitario panhispánico
}

\section{Analysis of the educational and social use of digital devices in the Panhispanic University context}

\author{
Esteban Vázquez Cano*, María Luisa Sevillano García* y Javier Fombona Cadavieco** \\ * Universidad Nacional de Educación a Distancia. España. \\ ** Universidad de Oviedo. España.
}

\begin{abstract}
Resumen
El objetivo de esta investigación ha sido analizar de forma general y comparada entre España e Hispanoamérica el uso que los estudiantes universitarios realizan de los dispositivos digitales móviles con fines educativos y sociales. Se analiza una muestra total de 886 estudiantes (442 españoles y 444 hispanoamericanos) correspondientes a cinco instituciones universitarias españolas y cinco hispanoamericanas. La metodología de investigación se desarrolla a través del análisis factorial y la comparación inter-grupos mediante pruebas no paramétricas. Los resultados generales en ambas zonas geográficas muestran que el uso educativo se concentra principalmente en el empleo de la tableta y el ordenador portátil para la búsqueda de información académica, el estudio y la consulta de servicios universitarios. Asimismo, las diferencias más significativas entre estudiantes españoles e hispanoamericanos muestran que en España se utiliza más la tableta y el "smartphone" para el estudio y en Hispanoamérica, el ordenador portátil.

Palabras clave: dispositivos digitales; ubicuidad; movilidad; educación superior.
\end{abstract}

\begin{abstract}
The objective of this research is to analyze from a general and comparative perspective how University students use mobile digital devices for educational and social purposes in Spain and
\end{abstract}

Correspondencia: Esteban Vázquez Cano, Facultad de Educación (UNED), Dpto. Didáctica. Organización Escolar y Didácticas Especiales. C/Juan del Rosal, 14 (28040) Madrid. E-mail:evazquez@edu.uned.es 
Latin America. It has been analysed a total sample of 886 students (442 from Spain and 444 from Latin America) from five Spanish Universities and five Latin American ones. The research methodology was based on factorial analysis and comparison between groups with non-parametric tests. The general results in both regions show that the educational purpose focuses on the use of tablets and laptops for searching academic information, studying and looking for information about University services. Likewise, the most significant differences between Spanish and Latin American students show that in Spain tablets and smartphones are more frequently used for educational purposes, but Latin America students use more frequently laptops.

Keywords: digital devices; ubiquity; mobility; higher education.

\section{Introducción}

Los dispositivos digitales móviles (teléfonos móviles inteligentes "smartphones", tabletas y ordenadores portátiles) son a la vez un instrumento de interacción social, un recurso didáctico individual y en el contexto de la Educación Superior pueden suponer un valioso aporte para desarrollar y fomentar nuevos modelos didácticos de enseñanza-aprendizaje (Franklin, 2011; Johnson, Adams Becker, Estrada, \& Freeman, 2014; Oulasvirta, Wahlström \& Ericsson, 2011; UNESCO, 2013; Vázquez-Cano, 2014). Estudios recientes muestran que tanto los modelos pedagógicos más tradicionales de la enseñanza presencial como la enseñanza a distancia mediada por los Entornos Virtuales de Aprendizaje (EVA) pueden verse mejorados con el uso de estos dispositivos dentro y fuera del recinto universitario (Ahmed \& Parsons, 2013; Burbules, 2012; Fombona, Pascual y Amador, 2012; Sevillano y Vázquez-Cano, 2015; Vázquez-Cano y Sevillano, 2014;). Asimismo, el contexto actual de la Educación Superior insta a las instituciones universitarias a promover una serie de competencias genéricas y específicas, donde el uso de estos dispositivos de forma personal, académica y profesional adquiere un destacado valor en el Espacio Europeo de Educación Superior (EEES); además de suponer un enriquecimiento de la práctica educativa universitaria (Johnson et al., 2014; Johnson, Adams Becker, Estrada, \& Freeman, 2015; UNESCO, 2013).

En este artículo presentamos un estudio del uso didáctico y social que realizan los estudiantes universitarios hispanoamericanos y españoles en 10 universidades de ambas zonas geográficas con el fin de establecer patrones de uso comunes y divergentes de forma que se puedan extrapolar conclusiones útiles para mejorar el contexto formativo de la Educación Superior en el mundo hispano.

\section{La sociedad digital: aprendizaje móvil y ubicuo mediado por dispositivos móviles}

El aprendizaje móvil y ubicuo hace referencia a las posibilidades que la tecnología móvil ofrece para el desarrollo de actividades de enseñanza-aprendizaje dentro y fuera de las aulas (Bedall-Hill, Jabbar \& Al Sheri, 2011; El-Hussein \& Cronje, 2010). El aprendizaje ubicuo es un nuevo paradigma educativo en el que el estudiante se posiciona ante el aprendizaje desde una perspectiva más global y en donde el espacio físico no es una variable determinante para su aprendizaje (Cope \& Kalantzis, 2009; García Mendoza, 2014; Sevillano y Vázquez-Cano, 2015). Los ambientes y lugares no formales -el café, la calle, los medios de transporte, el hogar, la red social, el ambiente de juego, los 
medios de comunicación y la cultura popular, el lugar de trabajo, etc.- se convierten en nuevos escenarios de aprendizaje (Barbosa, Barbosa \& Wagner, 2012; Keengwe, 2015; UNESCO, 2013). A este tipo de sociedad se le denomina "Sociedad de la ubicuidad" (Islas-Carmona, 2008). Este término designa una sociedad en la que cualquier persona puede disfrutar, en cualquier momento y en cualquier lugar, de una amplia gama de servicios a través de diversos dispositivos terminales y redes de banda ancha. Su lema es "anyone, anywhere, anytime" (cualquiera, en cualquier lugar y tiempo).

En los últimos años, el crecimiento de los dispositivos digitales móviles es constante y exponencial. Encabeza este ranking Hispanoamérica con un incremento del tráfico de datos móviles del 133\% en el último año 2014 y le sigue Europa con un incremento del 98\%. Las subscripciones de dispositivos móviles mundiales alcanzaron 7,1 billones en 2014 y se estima que en 2020 serán de 9,5 billones (Marketing Cloud, 2014). Un ejemplo claro de esta tendencia se produce en Hispanoamérica donde el primer semestre de 2014 en un estudio que abarcaba a más de 50 millones de usuarios, el uso de los ordenadores cayó un 11,3\%, mientras que el uso de smartphones creció un 70,1\% y las tabletas el 32\%. Los estudios realizados demuestran que el uso de dispositivos digitales móviles se consolida fuertemente y representa un 25,9\% del tráfico total anual de datos de Hispanoamérica (Invasión Mobile, 2014). Asimismo, en España la penetración y uso de smartphones y tabletas es muy alta. Más de 20 millones de españoles se conectan a internet a través del smartphone. El usuario es multipantalla: un 98\% de los usuarios utiliza diversos dispositivos en el mismo día, y un 90\% utiliza diferentes pantallas de modo secuencia. 36 millones de españoles (89\%) mayores de 13 años poseen un teléfono móvil y de ellos, más de 20 millones utilizan móviles inteligentes. Esto sitúa a España como el país europeo donde más se han expandido este tipo de terminales con una penetración del 118,2\%. Por primera vez, el smartphone se posiciona como el medio preferido para acceder a internet en España (85,5\% de los usuarios). También las tabletas ganan terreno en España, donde un $43 \%$ de los usuarios ya posee una, y un $45 \%$ de estos asegura usarla diariamente (Ditrendia, 2014). Las principales actividades que el público en general realiza con sus dispositivos móviles se centran en la consulta del correo electrónico en smartphones al menos una vez al día (91\%), la utilización de servicios de mensajería tipo Whatsapp principalmente desde un smartphone para mandar mensajes, al menos, una vez al día (90\%). La redacción de correos electrónicos (69\%) y la búsquedas en internet (70\%) se realizan mayoritariamente desde tabletas. Las tabletas se utilizan con mayor frecuencia en lugar de los smartphones para actividades en contextos menos dinámicos como el visionado de vídeos (40\%) y la lectura (57\%) (ADOBE, 2014; Deloitte, 2014). Estos datos mundiales y específicos para España e Hispanoamérica deben interpretarse desde la oportunidad de integrar estos dispositivos de una manera creativa y funcional en los procesos de enseñanza-aprendizaje que tienen lugar tanto en la educación media como en las instituciones educativas de la Educación Superior.

El gran potencial de los dispositivos digitales ha sido ya puesto de relevancia en numerosos estudios (Delfino, Dettori \& Lupi, 2009; Frohberg, Göth \& Schwabe, 2009). Se considera el aprendizaje móvil como una nueva vía de distribución de contenido (Muyinda, Lubega, \& Lynch, 2010), un activador de procesos reflexivos (VázquezCano, 2012; Vázquez-Cano, Fombona y Fernández, 2013) y como una nueva forma de integrar la gamificación y la realidad virtual en los procesos de enseñanza (Dahlstorm 
\& Warraich, 2013; Fombona, Goulao y García, 2014). El nuevo contexto de la Educación Superior basada en el desarrollo de competencias genéricas y específicas de los estudios universitarios insta a las instituciones del Espacio Europeo de Educación Superior (EEES) a desarrollar infraestructuras y modelos didácticos que favorezcan la asimilación de estas competencias consideradas necesarias para un desarrollo efectivo del egresado tanto social y académicamente como en su futuro profesional (Centeno Moreno, y Cubo Delgado, 2013; Maquilón Sánchez, Mirete Ruiz, García Sánchez, y Hernández Pina, 2013; Sánchez Vera, Prendes Espinosa, y Fernández Breis, 2013). Recientes informes internacionales muestran que los estudiantes universitarios creen que el uso de las tabletas transformará la forma en la que se estudiará en el futuro (81\%) y ayudarán a un aprendizaje más efectivo $(66 \%)$, y a mejorar su rendimiento académico en clase (62\%). De hecho, durante un día normal de estudio, los estudiantes utilizan asiduamente un ordenador portátil (72\%), el smartphone (37\%) y la tableta (21\%) (Poll, 2014). El desarrollo de una adecuada competencia digital está contemplado en los diferentes informes europeos e internacionales (Dublin Descriptors, 2004; Johnson et al., 2014, 2015; UNESCO, 2013); y para ello, el manejo contextualizado y aplicado de los dispositivos móviles y sus aplicaciones es una necesidad del estudiante y profesional del siglo XXI, y por ende, de las instituciones educativas que imparten esas competencias (Vladar \& Fife, 2010). El uso de dispositivos digitales en la Universidad puede ayudar a desarrollar de forma más efectiva el contenido teórico y hacerlo más práctico y colaborativo, fomentando el aprendizaje adaptativo e interactivo (Kinsella, 2009). Este tipo de funcionalidad puede desarrollarse a partir de aplicaciones de realidad aumentada, con minivídeos de contenido específico, con el desarrollo y diseño de apps modulares para las asignaturas universitarias, con el uso educativo de las redes sociales y el microblogging, entre otras actividades.

Por este motivo, muchas universidades a nivel mundial han empezado a promover el aprendizaje móvil con el uso de dispositivos digitales dentro y fuera de los campus universitarios. Por ejemplo, los estudiantes de la Universidad de Phoenix (Arizona) utilizan una app creada por la institución que les permite tener acceso a los materiales de las asignaturas, a foros temáticos en línea y fuera de línea y a participar en chats académicos desde cualquier lugar. En línea con estas iniciativas, la Universidad de Stanford (California) ofrece además de diferentes apps, un programa que se denomina: SMILE (Stanford Mobile Inquiry Learning Environment) y que permite a los estudiantes a través de sus dispositivos, crear, colaborar y evaluar preguntas relacionadas con temas educativos. En la Universidad Internacional de Florida (EEUU), los estudiantes disponen de una app que les permite consultar la disponibilidad de los fondos bibliográficos de la biblioteca y acceder a contenido audiovisual relacionado con las asignaturas. En línea con esta tendencia, el último Informe Horizon (2015) apuesta por la puesta en práctica de la tecnología "Wearable", el aprendizaje adaptado, el "Internet de las cosas" y la necesidad de adaptación tecnológica de las instituciones de Educación Superior para dar cabida a las iniciativas educativas BYOD (Bring Your Ownd Device-Trae tu propio dispositivo). Unas iniciativas que ya están tomando algunas universidades; por ejemplo, la Brunel University en Londres, la University of Western Australia y el propio King's College de Londres que ha renovado su infraestructura para dar soporte a la demanda de conexión mediante "BYOD" entre sus más de 6000 empleados y casi 
23.500 estudiantes. Universidades como la University System of Georgia (EEUU) ha desarrollado normativa específica para dar soporte a las iniciativas BYOD y la Ryerson University (Canada) ha mejorado los procesos de seguridad y privacidad para dar soporte en sus campus universitarios a estas iniciativas. Otras universidades como la Northern Illinois University (EEUU) imparten cursos a sus alumnos para utilizar de forma educativa sus propios dispositivos digitales.

Ante este contexto socio-digital que impregna casi todas las edades y estratos sociales, las implicaciones del uso educativo y social de los dispositivos digitales móviles pueden tener hondas repercusiones para entender los nuevos patrones de uso de estos dispositivos en el aprendizaje, para adecuar y mejorar la infraestructura tecnológica y espacial de los campus universitarios, para favorecer modelos de interactividad grupal en el estudio, para la implementación de contenidos adecuados al lugar y necesidad del estudiante, para la adecuación del formato del contenido educativo a los diferentes dispositivos y, en definitiva, para dar una mejor respuesta tecnológica, de contenido y social al estudiante que hace uso de los dispositivos digitales móviles como un recurso más para el estudio y la interacción social desde múltiples localizaciones.

\section{Método}

El objetivo de este estudio es comprobar qué tipo de actividades y procesos realizan los estudiantes universitarios con dispositivos digitales móviles en el ámbito académico y social y si se pueden establecer diferencias significativas entre el contexto español e hispanoamericano. Los participantes conforman una muestra total de 886 estudiantes universitarios (442 españoles y 444 hispanoamericanos) correspondientes a cinco universidades españolas y cinco hispanoamericanas según la muestra de la Tabla 1.

Tabla 1

Universidades participantes

\begin{tabular}{|c|c|c|}
\hline Universidades & & N. ${ }^{\circ}$ protocolos \\
\hline \multicolumn{3}{|l|}{ España } \\
\hline Madrid. Universidad Complutense de Madrid & & 42 \\
\hline Vigo. Universidad de Vigo & & 46 \\
\hline Oviedo. Universidad de Oviedo & & 169 \\
\hline Granada. Universidad de Granada & & 77 \\
\hline \multirow[t]{2}{*}{ Madrid. Universidad Nacional de Educación a Distancia (UNED) } & & 108 \\
\hline & Total & 442 \\
\hline \multicolumn{3}{|l|}{ Hispanoamérica } \\
\hline Chile. Universidad del Libertador Bernardo O'higgins & & 98 \\
\hline Perú. Universidad Nacional Hermilio Valdizán. Huánuco & & 52 \\
\hline Colombia. Universidad de Cartagena & & 110 \\
\hline Panamá. Universidad Pública de Panamá & & 79 \\
\hline \multirow[t]{2}{*}{ México. Universidad Veracruzana. Xalapa } & & 105 \\
\hline & Total & 444 \\
\hline
\end{tabular}


La muestra obtenida por edades y diferenciada por zona geográfica (España/Hispanoamérica) se presenta en la Tabla 2.

Tabla 2

Muestra según la edad

\begin{tabular}{|c|c|c|c|c|c|}
\hline \multicolumn{3}{|c|}{$\begin{array}{c}\text { España } \\
\text { Hispanoamérica }\end{array}$} & \multicolumn{2}{|c|}{ Zona geográfica } & \multirow{2}{*}{$\begin{array}{c}\text { Total } \\
236\end{array}$} \\
\hline \multirow{9}{*}{ Edad } & \multirow{2}{*}{$18-20$} & Recuento & 108 & 128 & \\
\hline & & $\%$ del total & $12,1 \%$ & $14,4 \%$ & $26,6 \%$ \\
\hline & \multirow{2}{*}{$21-23$} & Recuento & 146 & 151 & 297 \\
\hline & & $\%$ del total & $16,4 \%$ & $17,0 \%$ & $33,5 \%$ \\
\hline & \multirow{2}{*}{$24-27$} & Recuento & 44 & 69 & 113 \\
\hline & & $\%$ del total & $4,9 \%$ & $7,7 \%$ & $12,7 \%$ \\
\hline & \multirow{2}{*}{$28-31$} & Recuento & 27 & 54 & 81 \\
\hline & & $\%$ del total & $3,0 \%$ & $6,0 \%$ & $9,1 \%$ \\
\hline & \multirow{2}{*}{ más de 31} & Recuento & 119 & 42 & 151 \\
\hline \multirow{3}{*}{ Total } & & $\%$ del total & $13,4 \%$ & $4,7 \%$ & $18,1 \%$ \\
\hline & & Recuento & 442 & 444 & 886 \\
\hline & $\%$ del total & $49,8 \%$ & $50,1 \%$ & $100,0 \%$ & \\
\hline
\end{tabular}

La muestra por sexo es equitativa entre las dos zonas geográficas (España 51\% e Hispanoamérica 49\%). En la Tabla 3, se puede comprobar la distribución porcentual parcial y acumulada según el sexo de los encuestados.

Tabla 3

Distribución de la muestra según el sexo

\begin{tabular}{|c|c|c|c|c|c|}
\hline \multicolumn{3}{|c|}{$\begin{array}{c}\text { España } \\
\text { Hispanoamérica }\end{array}$} & \multicolumn{2}{|c|}{ Zona geográfica } & \multirow{2}{*}{$\begin{array}{c}\text { Total } \\
250\end{array}$} \\
\hline & Hombro & Recuento & 108 & 142 & \\
\hline & Hombre & $\%$ del total & $12,1 \%$ & $16,0 \%$ & $28,2 \%$ \\
\hline \multirow{2}{*}{ Sexo } & \multirow{2}{*}{ Mujer } & Recuento & 334 & 302 & 636 \\
\hline & & $\%$ del total & $37,6 \%$ & $34,0 \%$ & $71,7 \%$ \\
\hline \multirow[t]{2}{*}{ Total } & & Recuento & 442 & 444 & 886 \\
\hline & $\%$ del total & $51,0 \%$ & $49,0 \%$ & $100,0 \%$ & \\
\hline
\end{tabular}

La investigación se desarrolló durante dos cursos académicos (2012/13 y 2013/14) con estudiantes de carreras universitarias de Ciencias Sociales en los cursos segundo y tercero de forma que la muestra fuera lo más homogénea posible para poder establecer criterios comparativos en el análisis. Esta investigación se enmarca dentro del Plan 
Nacional I+D+I (Aprendizaje ubicuo con dispositivos móviles: elaboración y desarrollo de un mapa de competencias en Educación Superior). En una primera fase durante el curso académico 2012/13 se diseñó y validó un cuestionario registrado en la Oficina de Patentes y Marcas de España con número de marca: 3.502-443 y denominación "MAUDIMO". En el desarrollo de este cuestionario han intervenido diez profesores universitarios (5 Hispanoamericanos y 5 Españoles correspondientes a cada una de las Universidades implicadas en la investigación). Una parte de este cuestionario estaba compuesta por tres macrocategorías correspondientes a tres dispositivos digitales: tableta, samrtphone y ordenador portátil sobre los que se ha preguntado en una escala Likert (1 nada/5 mucho) sobre el uso académico y social con referencia a los siguientes ítems: elaboración de trabajos académicos, búsqueda de información académica, estudio, intercambio de apuntes, coordinación de trabajos grupales con compañeros, consulta de servicios universitarios, búsqueda de información no académica, chat y mensajería instanánea, correo electrónico y redes sociales. En la segunda fase - desarrollada durante el curso 2013/14 - se procedió a la realización del análisis estadístico a través de un método mixto factorial (Bonett \& Price, 2005) que ha tenido en cuenta que la correlación entre dos ítems o variables del cuestionario depende de su similitud sustantiva (el contenido del ítem), pero también de las semejanzas de sus distribuciones estadísticas (Bernstein, Garbin \& Teng, 1988, p. 398). Esto significa que ítems con distribuciones similares correlacionarán con mayor intensidad que con aquéllos con distribuciones diferentes (McLeod, Swygert \& Thissen, 2001). Por ejemplo, ítems fáciles de responder se agruparán frente a ítems más difíciles, aún cuando todos los ítems midan la misma variable latente (Nunnaly \& Bernstein, 1994, p. 318).

Aplicar un análisis factorial sin antes cerciorarnos de que este no es el caso puede producir factores basados solamente en la semejanza de distribuciones y no en una verdadera variable latente que sustantivamente resuma dichos ítems o variables (Ferrando, 2009; Hair, Anderson, Tatham \& Black, 1988).

Por lo tanto, hemos realizado un primer análisis factorial con el programa Factor 9 para generar los factores más representativos del uso ubicuo de los dispositivos digitales móviles en el total de la muestra (España e Hispanoamérica). Posteriormente, hemos comprobado si los factores obtenidos tenían distribuciones normales para calcular posibles diferencias inter-grupos. La normalidad se ha calculado mediante la prueba de "Kolmogorov-Smirnov" y los resultados de normalidad de los factores nos han permitido analizar con pruebas no paramétricas (U Mann Whitney) la diferencia inter-grupos para confrontar los resultados de las dos zonas geográficas.

\section{Resultados}

Primeramente, hemos evaluado la fiabilidad del cuestionario empleado mediante el test de esfericidad de Bartlett y la prueba de adecuación muestral KMO (Tabla 4).

La significación para la prueba de Bartlett $(\mathrm{p}<0.05)$ indica que nuestra matriz es distinta de la matriz unidad con un nivel de confianza del 95\%, y que por tanto, existen correlaciones significativas entre las variables que apuntan a la posible existencia de variables latentes - los factores - que las expliquen. Por su parte, el test de adecuación muestral KMO arroja un valor cercano a 1 (0.893), por lo que las correlaciones 
parciales de nuestras variables son muy pequeñas. Asimismo, el resultado del Alfa de Cronbach fue 0.876. Adoptamos el método de ejes principales como el mejor para desentrañar la estructura latente que buscamos en las variables (Bartholomew, 1987). Es decir, puesto que trabajamos con variables tipificadas (matriz de correlaciones y no de covarianzas), sus varianzas son siempre 1. Se realiza con el programa Factor un análisis paralelo del tipo "optimal implementation" para la determinación final del número de factores. De todos ellos, los 5 primeros cumplen con el criterio de tener autovalores mayores que 1 y representan una varianza total explicada del 70,1\%. Los autovalores se detallan en la Tabla 4.

Tabla 4

Adecuación de la Matriz de Correlaciones

\begin{tabular}{ll}
\hline Determinante de la matriz & 0.000000004350151 \\
\hline Estadístico de Bartlett & $13262.1(\mathrm{gl}=1035 ; \mathrm{P}=0.000010)$ \\
Test de Kaiser-Meyer-Olkin $(\mathrm{KMO})$ & 0.89333 \\
\hline
\end{tabular}

Tabla 4

Varianza total explicada basada en los autovalores

\begin{tabular}{cccc}
\hline Variable & Autovalor & Proporción de Varianza & Proporción Acumulada de Varianza \\
\hline 1 & 8.13037 & 20,675 & 20,675 \\
2 & 7.48745 & 18,755 & 39,430 \\
3 & 2.78812 & 16,061 & 55,491 \\
4 & 1.91387 & 8,161 & 63,652 \\
5 & 1.62402 & 6,530 & 70,182 \\
\hline
\end{tabular}

En estas circunstancias, se lleva a cabo la rotación oblicua de los factores para su interpretación. Ese cambio de ejes nos ayuda a separar y discriminar mejor cómo se relacionan con ellos las variables. A continuación, incluimos la matriz de pesos factoriales para los factores extraídos y rotados (eliminando aquéllos que no superan el valor de 0,30) (Tabla 5).

La interpretación de los cinco factores conforme a su varianza total y a la incidencia significativa en ambos grupos geográficos es la siguiente:

Factor 1. Uso educativo de la tableta:

- V2. Búsqueda de información académica $(0,876)$.

- V3. Estudio (0,785).

- V6. Consulta servicios universitarios $(0,801)$. 
Tabla 5

Varianza total explicada

\begin{tabular}{|c|c|c|c|c|c|}
\hline Variables & F1 & F2 & F3 & F4 & F5 \\
\hline \multicolumn{6}{|l|}{ V1. Tableta. Elaboración de trabajos académicos } \\
\hline V2. Tableta. Búsqueda de información académica & 0.876 & & & & \\
\hline V3. Tableta. Estudio & 0.785 & & & & \\
\hline \multicolumn{6}{|l|}{ V4. Tableta. Intercambio de información académica } \\
\hline \multicolumn{6}{|l|}{ V5. Tableta. Coordinación de trabajos grupales } \\
\hline V6. Tableta. Consulta servicios universitarios & 0.801 & & & & \\
\hline V7. Tableta. Búsq. de información no académica & & & & & 0.556 \\
\hline V8. Tableta. Chat y mensajería instantánea & & & & & 0.682 \\
\hline V9. Tableta. Correo electrónico & & & & & 0.820 \\
\hline V10. Tableta. Redes sociales & & & & & 0.975 \\
\hline \multicolumn{6}{|l|}{ V11. Smartphone. Elaboración de trabajos académicos } \\
\hline \multicolumn{6}{|l|}{ V12. Smartphone. Búsqueda de información académica } \\
\hline \multicolumn{6}{|l|}{ V13. Smartphone. Estudio } \\
\hline V14. Smartphone. Intercambio de información académica & & & & 0.869 & \\
\hline V15. Smartphone. Coordinación de trabajos grupales & & & & 0.675 & \\
\hline V16. Smartphone. Consulta servicios universitarios & & & & 0.869 & \\
\hline \multicolumn{6}{|l|}{ V17. Smartphone. Búsq. de información no académica } \\
\hline V18. Smartphone. Chat y mensajería instantánea & & & 0.975 & & \\
\hline V19. Smartphone. Correo electrónico & & & 0.583 & & \\
\hline V20. Smartphone. Redes sociales & & & 0.784 & & \\
\hline V21. Portátil. Elaboración de trabajos académicos & & 0.818 & & & \\
\hline V22. Portátil. Búsqueda de información académica & & 0.776 & & & \\
\hline V23. Portátil. Estudio & & 0.638 & & & \\
\hline \multicolumn{6}{|l|}{ V24. Portátil. Intercambio de información académica } \\
\hline \multicolumn{6}{|l|}{ V25. Portátil. Coordinación de trabajos grupales } \\
\hline V26. Portátil. Consulta servicios universitarios & & 0.689 & & & \\
\hline \multicolumn{6}{|l|}{ V27. Portátil. Búsq. de información no académica } \\
\hline \multicolumn{6}{|l|}{ V28. Portátil. Chat y mensajería instantánea } \\
\hline \multicolumn{6}{|l|}{ V29. Portátil. Correo electrónico } \\
\hline V30. Portátil. Redes sociales & & & & & \\
\hline
\end{tabular}

El Factor 1 representa una varianza total del 20,675\% y muestra resultados significativos del uso educativo de la tableta entre los estudiantes españoles e hispanoamericanos para usos educativos. Especialmente relevante es el uso de este dispositivo para la búsqueda de información académica $(, 876)$, para el estudio $(, 785)$ y la consulta de servicios universitarios $(, 801)$. 
Factor 2. Uso educativo del ordenador portátil:

- V21. Elaboración de trabajos académicos $(0,818)$.

- V22. Búsqueda de información académica $(0,776)$.

- V23. Estudio (0,638).

- V26. Consulta servicios universitarios $(0,689)$.

El Factor 2 acumula una varianza del 18,755\% y muestra la incidencia del uso educativo del smartphone por parte del estudiante universitario. Es significativo que se use el ordenador portátil para la elaboración de trabajos académicos $(0,818)$. En segundo lugar, su uso se centra en el búsqueda de información académica $(0,776)$ y, por último, en el estudio $(0,638)$ y la consulta de servicios académicos $(0,689)$.

Factor 3. Uso social del smartphone:

- V18. Chat y mensajería instantánea $(0,975)$.

- V19. Correo electrónico $(0,583)$.

- V20. Redes sociales $(0,784)$.

El Factor 3 desciende significativamente su impacto en la varianza explicada $(16,061 \%)$ y muestra el uso social del smartphone. El uso educativo de este dispositivo tiene un uso muy extendido entre el estudiante universitario español e hispanoamericano. Su uso se concentra en el uso de chat y mensajería instantánea $(0,975)$ y en la interacción en redes sociales $(0,784)$ y la consulta del correo electrónico $(0,583)$.

Factor 4. Uso educativo del smartphone:

- V14. Intercambio de información académica $(0,869)$.

- V15. Coordinación de trabajos grupales $(0,675)$.

- V16. Consulta servicios universitarios $(0,869)$.

El Factor 4 representa un 8,161\% del total de la varianza explicada y corresponde con el uso educativo del smartphone. El uso educativo principal se desarrolla en el ámbito del trabajo en grupo y colaborativo, lo que representa un aspecto muy positivo para el fomento de competencias transversales. Es significativo que se utilice este dispositivo para intercambiar información académica entre los estudiantes $(0,869)$ y para coordinar trabajos en grupo $(0,675)$.

Factor 5. Uso social de la tableta:

- V7. Búsqueda de información no académica $(0,556)$.

- V8. Chat y mensajería instantánea $(0,682)$.

- V9. Correo electrónico $(0,820)$.

- V10. Redes sociales $(0,975)$.

El Factor 5 representa un 6,530\% del total de la varianza explicada y, aunque existen varios ítems implicados, tienen una baja significación. El uso social de la tableta tiene relación con actividades comunicativas: mensajería y chat $(0,682)$, correo electrónico $(0,820) \mathrm{y}$, principalmente, con la interacción en redes sociales $(0,975)$. 
A continuación, hemos comprobado si los factores obtenidos tienen distribuciones normales para calcular posteriormente si existen diferencias inter-grupos. Para probar la normalidad hemos aplicado la prueba "Kolmogorov-Smirnov" (Tabla 6).

Tabla 6

Prueba de Kolmogorov-Smirnov para una muestra

\begin{tabular}{llccccc}
\hline & & Factor 1 & Factor 2 & Factor 3 & Factor 4 & Factor 5 \\
\hline $\mathrm{N}$ & & 886 & 886 & 886 & 886 & 886 \\
Parámetros & Media &, 00002 &, 00001 &, 00001 &, 00001 &,- 00001 \\
normales $^{\mathrm{a}, \mathrm{b}}$ & Desviación típica & 1,000700 & 1,000707 & 1,000711 & 1,000713 & 1,000712 \\
& Absoluta &, 353 &, 293 &, 118 &, 089 &, 242 \\
Diferencias más & Positiva &, 353 &, 293 &, 118 &, 089 &, 190 \\
extremas & Negativa &,- 243 &,- 202 &,- 089 &,- 050 &,- 242 \\
& 9,374 & 7,787 & 3,133 & 2,352 & 6,424 \\
Z de Kolmogorov-Smirnov &, 000 &, 000 &, 000 &, 000 &, 000 \\
\multicolumn{2}{l}{ Sig. asintót. (bilateral) } & & & & & \\
\hline
\end{tabular}

Los cinco primeros factores tienen distribuciones no normales por lo que aplicamos sucesivas pruebas U de Mann-Whitney (Tabla 7) para comprobar si existen diferencias significativas inter-grupos: españoles e hispanoamericanos.

Tabla 7

Estadísticos de contraste Mann-Whitney

Estadísticos de contraste ${ }^{\mathrm{a}}$

\begin{tabular}{lccccc} 
& Factor 1 & Factor 2 & Factor 3 & Factor 4 & Factor 5 \\
\hline U de Mann-Whitney & 46418,500 & 55424,000 & 58107,500 & 44577,000 & 61352,000 \\
W de Wilcoxon & 100703,500 & 126677,000 & 129360,500 & 115830,000 & 115637,000 \\
Z & $-5,770$ & $-2,439$ & $-1,446$ & $-6,451$ &,- 246 \\
Sig. asintót. (bilateral) &, 000 &, 015 &, 148 &, 000 &, 806 \\
\hline
\end{tabular}

a. Variable de agrupación: País (muestra nacional y extranjera sin desagregar)

Podemos comprobar cómo los factores 1, 2 y 4 muestran diferencias entre los dos grupos geográficos. Los resultados inter-grupos han sido los siguientes:

Para el Factor 1: hay diferencias significativas entre ambos grupos: $U(704)=46418.5$, $\mathrm{p}<0,001$

Para el Factor 2: hay diferencias significativas entre ambos grupos: $U(704)=55424.0$, $\mathrm{p}=0.015$ 
Para el Factor 3: no hay diferencias significativas entre ambos grupos: $U(704)=$ 58107.5, $\mathrm{p}=0.148$

Para el Factor 4: hay diferencias significativas entre ambos grupos: $U(704)=44577.0$, $\mathrm{p}<0.001$

Para el Factor 5: no hay diferencias significativas entre ambos grupos: $U(704)=$ 61352.0, $\mathrm{p}=0.806$

Para comprobar estas diferencias recurrimos a una tabla de contingencia que nos permita observar las diferencias desagradas por zonas geográficas y países (Tabla 8).

Tabla 8

Tabla de contingencia por factores desagregada por países.

\begin{tabular}{|c|c|c|c|c|c|c|}
\hline \multirow[b]{2}{*}{$\begin{array}{l}\text { Países } \\
\text { Nada }\end{array}$} & \multicolumn{5}{|c|}{ Factor 1. Uso educativo de la tableta } & \multirow[b]{2}{*}{ Total } \\
\hline & $\begin{array}{l}\text { Casi } \\
\text { nada }\end{array}$ & $\begin{array}{c}\text { De } \\
\text { vez en } \\
\text { cuando }\end{array}$ & A menudo & \multicolumn{2}{|l|}{ Siempre } & \\
\hline España & $16,4 \%$ & $18,1 \%$ & $18,5 \%$ & $29,0 \%$ & $18,0 \%$ & $100,0 \%$ \\
\hline Colombia & $40,0 \%$ & $20,7 \%$ & $19,9 \%$ & $9,3 \%$ & $10,1 \%$ & $100,0 \%$ \\
\hline Panamá & $31,4 \%$ & $25,6 \%$ & $21,6 \%$ & $12,1 \%$ & $9,3 \%$ & $100,0 \%$ \\
\hline Perú & $35,3 \%$ & $19,9 \%$ & $22,7 \%$ & $13,1 \%$ & $9,0 \%$ & $100,0 \%$ \\
\hline México & $30,7 \%$ & $18,7 \%$ & $31,1 \%$ & $11,3 \%$ & $8,2 \%$ & $100,0 \%$ \\
\hline Chile & $29,6 \%$ & $23,1 \%$ & $28,1 \%$ & $9,9 \%$ & $9,3 \%$ & $100,0 \%$ \\
\hline \multirow[b]{2}{*}{$\begin{array}{l}\text { Países } \\
\text { Nada }\end{array}$} & & actor $2 . \mathrm{l}$ & educativo d & denador $p$ & & \multirow[b]{2}{*}{ Total } \\
\hline & $\begin{array}{c}\text { Casi } \\
\text { nada }\end{array}$ & $\begin{array}{c}\text { De } \\
\text { vez en } \\
\text { cuando }\end{array}$ & A menudo & Siempre & & \\
\hline España & $14,1 \%$ & $25,9 \%$ & $27,8 \%$ & $13,1 \%$ & $19,1 \%$ & $100 \%$ \\
\hline Colombia & $7,8 \%$ & $19,9 \%$ & $23,1 \%$ & $21,2 \%$ & $28,0 \%$ & $100 \%$ \\
\hline Panamá & $23,8 \%$ & $11,9 \%$ & $23,1 \%$ & $21,1 \%$ & $20,1 \%$ & $100 \%$ \\
\hline Perú & $14,9 \%$ & $19,1 \%$ & $25,6 \%$ & $20,9 \%$ & $19,5 \%$ & $100 \%$ \\
\hline México & $5,7 \%$ & $17,1 \%$ & $26,4 \%$ & $24,9 \%$ & $25,9 \%$ & $100 \%$ \\
\hline Chile & $23,9 \%$ & $15,1 \%$ & $26,7 \%$ & $14,8 \%$ & $19,5 \%$ & $100 \%$ \\
\hline \multirow[b]{2}{*}{$\begin{array}{l}\text { Países } \\
\text { Nada }\end{array}$} & & Factor & Iso educativ & l smartph & & \multirow[b]{2}{*}{ Total } \\
\hline & $\begin{array}{l}\text { Casi } \\
\text { nada }\end{array}$ & $\begin{array}{c}\text { De } \\
\text { vez en } \\
\text { cuando }\end{array}$ & A menudo & Siempre & & \\
\hline España & $27,0 \%$ & $10,3 \%$ & $11,5 \%$ & $21,1 \%$ & $30,1 \%$ & $100 \%$ \\
\hline Colombia & $33,5 \%$ & $17,7 \%$ & $25,5 \%$ & $12,2 \%$ & $11,1 \%$ & $100 \%$ \\
\hline Panamá & $16,7 \%$ & $18,8 \%$ & $36,1 \%$ & $11,3 \%$ & $17,1 \%$ & $100 \%$ \\
\hline Perú & $31,8 \%$ & $18,6 \%$ & $25,6 \%$ & $9,9 \%$ & $14,1 \%$ & $100 \%$ \\
\hline México & $30,7 \%$ & $17,0 \%$ & $24,3 \%$ & $12,0 \%$ & $16,0 \%$ & $100 \%$ \\
\hline Chile & $29,4 \%$ & $19,8 \%$ & $21,5 \%$ & $16,8 \%$ & $12,5 \%$ & $100 \%$ \\
\hline
\end{tabular}


El Factor 1 (Uso educativo de la tableta) presenta diferencias significativas entre las dos zonas geográficas. En España se utiliza más la tableta para el estudio. El porcentaje acumulado de los estudiantes españoles que lo hacen "a menudo" o "siempre" es del $47 \%$, en comparación con otros países hispanoamericanos: Chile (19,2\%), Colombia (19,4\%), México (19,5\%), Panamá (21,4\%) y Perú (22,1\%). Asimismo, el Factor 2 (Uso educativo del ordenador portátil), presenta un mayor uso educativo en los estudiantes hispanoamericanos que los españoles en aspectos como la búsqueda de la información académica y el estudio. En estos dos aspectos, países como Colombia y México superan a España en una media de 13,9\%. El Factor 4 (Uso educativo del smartphone) también presenta diferencias porcentuales entre las dos zonas geográficas. Los estudiantes españoles emplean el smartphone en un porcentaje mucho mayor para el intercambio de información académica $(+12,3 \%)$ con respecto a la media del resto de países hispanoamericanos analizados.

\section{Discusión}

El objetivo de esta investigación es analizar el uso académico y social que los estudiantes universitarios españoles e hispanoamericanos realizan con sus dispositivos digitales móviles (smartphones, tabletas y ordenadores portátiles) y establecer posibles implicaciones educativas y diferencias entre los diferentes países.

Los resultados del análisis estadístico factorial global muestran que el uso educativo de los dispositivos digitales móviles en el mundo hispano se concentra principalmente en el uso de la tableta y el ordenador portátil para la búsqueda de información académica, el estudio y la consulta de servicios universitarios. Resultados similares han sido puestos de relieve en otras zonas geográficas como Japón (Ogata et al., 2014) y África (Muyinda, Lubega \& Lynch, 2010). El smartphone se utiliza de forma educativa para el intercambio de información académica y la coordinación de trabajos grupales y es el dispositivo sobre el que se están realizando más estudios como precursor y facilitador de procesos de enseñanza-aprendizaje ubicuos (Barbosa, Barbosa \& Wagner, 2012; Chen \& deNoyelles, 2013; Norris, Hossain \& Soloway, 2011). La UNESCO (2013) considera al smartphone una de las herramientas educativas con mayor proyección educativa en países en vías de desarrollo. Los dos dispositivos digitales que españoles e hispanoamericanos utilizan socialmente son el smartphone y la tableta, principalmente para chatear por mensajería instantánea, consultar el correo electrónico e interactuar en redes sociales. Estos resultados también han sido refrendados por los principales informes de uso realizados por diferentes compañías a nivel mundial (Ditrendia, 2014; Deloitte, 2014, Invasión Mobile, 2014; Marketing Cloud, 2014).

Los resultados de las sucesivas pruebas paramétricas para la comparación intergrupos en las dos zonas geográficas nos han permitido determinar si existen diferencias de uso en los diferentes países. El estadístico de contraste "Mann-Whitney" muestra diferencias significativas entre españoles e hispanoamericanos en tres factores: Factor 1. Uso educativo de la tableta, Factor 2. Uso educativo del ordenador portátil y Factor 4. Uso educativo del smartphone. Las diferencias más significativas nos permiten observar que en España se utiliza más la tableta para el estudio que en Hispanoamérica. Asimismo, los estudiantes hispanoamericanos utilizan más el ordenador portátil de 
forma educativa que los españoles, principalmente para la búsqueda de la información académica y para el estudio. Por último, el uso educativo del smartphone es el que presenta mayores diferencias porcentuales entre las dos zonas geográficas. Los estudiantes españoles emplean el smartphone en un porcentaje mucho mayor para el intercambio de información académica $(+12,3 \%)$ con respecto a la media del resto de países hispanoamericanos analizados.

Estos resultados muestran que los estudiantes universitarios en el mundo hispano realizan un uso diversificado tanto en el ámbito educativo como de interacción social. Esta situación debería instar a las instituciones, profesores y responsables educativos a una mejora constante de los procesos didácticos, de los contenidos y de los formatos en los que se ofrecen estos contenidos así como las formas de interacción y trabajo colaborativo. Además, el conocimiento del patrón de conexión conforme al espacio y lugar desde el que se conecta el dispositivo digital móvil de un estudiante con fines educativos puede aprovecharse para el diseño de actividades sensibles al contexto que enriquezcan la experiencia formativa y contextualicen el contenido teórico con recursos como la realidad aumentada o los grupos colaborativos en red. Las posibilidades son numerosas y diversas conforme al carácter de los estudios y las asignaturas implicadas, aunque las dificultades son también importantes, entre las que destacan, la privacidad de las comunicaciones, la formación del profesorado, la inversión en infraestructuras tecnológicas y la actualización hacia diseños tecno-didácticos avanzados que entronquen con la realidad social y profesional de la sociedad actual.

\section{Apoyos}

Este trabajo se enmarca en el Proyecto de la Dirección General de Investigación y Gestión del Plan Nacional I+D+I (Aprendizaje ubicuo con dispositivos móviles: elaboración y desarrollo de un mapa de competencias en educación superior) EDU201017420-Subprograma EDUC.

\section{Referencias}

ADOBE (2014). U.S. Mobile Benchmark Report. Recuperado de: http://www.cmo.com/ content/dam/CMO_Other/ADI/ADI_Mobile_Report_2014/2014_US_Mobile_Benchmark_Report.pdf

Ahmed, S., \& Parsons, D. (2013). Abductive science inquiry using mobile devices in the classroom. Computers \& Education, 63, 62-72.

Barbosa, J.L., Barbosa, D.N., \& Wagner, A. (2012). Learning in Ubiquitous Computing Environments. International Journal of Information and Communication Technology Education, 8(3), 64-77.

Bartholomew, D.J. (1987). Latent Variable Models and Factor Analysis. New York, NY: Oxford University Press.

Bedall-Hill, N., Jabbar, A., \& Al Sheri, S. (2011). Social mobile devices as tools for qualitative research in education: iPhones and iPads in ethnography, interviewing, and design-based research. Journal of the Research Centre for Educational Technology, 7, 67-89. 
Bernstein, I.H., Garbin, C., \& Teng, G. (1988). Applied Multivariate Analysis. New York, NY: SpringerVerlag.

Burbules, N. (2012). Ubiquitous Learning and the Future of Teaching. Encounters, 13, 3-14.

Centeno Moreno, G., \& Cubo Delgado, S. (2013). Evaluación de la competencia digital y las actitudes hacia las TIC del alumnado universitario. Revista de Investigación Educativa, 31(2), 517-536. Doi: http://dx.doi.org/10.6018/rie.31.2.169271

Chen, B., \& deNoyelles, A. (2013). Exploring Students' Mobile Learning Practices in Higher Education. Educase Review Online. Recuperado de http://www.educause.edu/ ero/article/exploring-students-mobile-learning-practices-higher-education

Cope, B., \& Kalantzis, M. (2009). Multiliteracies: New literacies, new learning, pedagogies. An International Journal, 4, 164-195.

Dahlstorm, E., \& Warraich, K. (2013). Student mobile computing practices, 2012: Lessons Learned from Qatar (Research Report). Louisville, CO: Educase Center for Applied Research. Recuperado de http://educase.edu/ecar

Deloitte (2014). Consumo Móvil en España 2014 Revolución y evolución. Recuperado de http://www2.deloitte.com/es/es/pages/technology-media-and-telecommunications/ articles/consumo-medios-espana.html

Delfino, M., Dettori, G., \& Lupi, V. (2009). Task-based learning and ICT: creative activities in the context of a European project. eLearning Papers, 16, 1-11.

Ditrendia (2014). Informe Ditrendia: Mobile en España y el mundo. Recuperado el 20 de http://www.ditrendia.es/wp-content/uploads/2014/07/Ditrendia-Informe-Mobile-enEspa\%C3\%B1a-y-en-el-Mundo.pdf

Dublin Descriptors (octubre, 2004). Shared "Dublin" descriptors for the bachelor's, master's and doctoral awards. Trabajo presentado en el Joint Quality Initiative, Dublin.

El-Hussein, M. O. M., \& Cronje, J. C. (2010). Defining Mobile Learning in the Higher Education Landscape. Educational Technology \& Society, 13(3), 12-21.

Fombona, J., Pascual, A., \& Amador, F. (2012). Realidad Aumentada, una evolución de las aplicaciones de los dispositivos móviles. PixelBit, 41, 197-210.

Fombona, J., Goulao, M. F., \& García, M. (2014). Melhorar a atratividade da informação a través do uso da realidade aumentada. Perspectivas em Ciencia da Informaçao, 19(1), 37-50

Frohberg, C., Göth, C., \& Schwabe G. (2009). Mobile Learning projects, a critical analysis of the state of the art. Journal of Computer Assisted Learning, 25(4), 307-331.

Garcia Mendoza, G.A. (2014). A comparative study of computer and mobile phonemediated collaboration: the case of university students in Japan. Mobile Learning Applications in Higher Education. RUSC. Universities And Knowledge Society Journal, 11(1), 222-237.

Hair, J. F., Anderson, R.E., Tatham, R. L., \& Black, W. C. (1988). Multivariate Data Analysis. New Jersey, NJ: Prentice Hall.

Johnson, L., Adams Becker, S., Estrada, V., \& Freeman, A. (2014). NMC Horizon Report: 2014 Higher Education Edition. Austin, TX: The New Media Consortium.

Johnson, L., Adams Becker, S., Estrada, V., \& Freeman, A. (2015). NMC Horizon Report: 2015 Higher Education Edition. Austin, TX: The New Media Consortium. 
Islas-Carmona, J.O. (2008). El prosumidor: El actor comunicativo de la sociedad de la ubicuidad. Palabra Clave, 11(1), 29-39.

Invasión Mobile (2014). Invasión Mobile en Latinoamérica. Recuperado de http://guialocal. com.ar/lp/informe/invasion-mobile-latam-2014/ES/invasion-mobile-latam-2014.html

Keengwe, J. (2015). Promoting active learning through the integration of mobile and ubiquitous technologies. Hershey, PA: IGI Global.

Kinsella, S. (2009). Many to One: Using the mobile phone to interact with large classes. British Journal of Education Technology, 40(5), 956-958.

Maquilón Sánchez, J., Mirete Ruiz, A., García Sánchez, F., \& Hernández Pina, F. (2013). Valoración de las TIC por los estudiantes universitarios y su relación con los enfoques de aprendizaje. Revista de Investigación Educativa, 31(2), 537-554. Doi: http:// dx.doi.org/10.6018/rie.31.2.151891

Marketing Cloud (2014). 2014 Mobile Behavior Report Combining mobile device tracking and consumer survey data to build a powerful mobile strategy. Recuperado de http://www. exacttarget.com/sites/exacttarget/files/deliverables/etmc-2014mobilebehaviorreport.pdf

McLeod, L.D., Swygert, K.A., \& Thissen, D. (2001). Factor analysis for items scored in two categories. En D. Thissen \& H. Wainer (Eds.), Test scoring (pp. 189-216). Mahwah, NJ: Lawrence Erlbaum.

Muyinda, P.B., Lubega, J.T., \& Lynch, K. (2010). Unleashing mobile phones for research supervision support at Makerere University, Uganda: the lessons learned. International Journal of Innovation and Learning, 7(1), 14-34.

Norris, C., Hossain, A., \& Soloway, E. (2011). Using smartphones as essential tools for learning: A call to place schools on the right side of the 21st century. Educational Technology, 51(3), 18-25.

Nunnaly, J., \& Bernstein, I. (1994). Psychometric Theory. New York, NY: McGraw-Hill.

Oulasvirta, A., Wahlström, M., \& Ericsson, K.A. (2011). What does it mean to be good at using a mobile device? An investigation of three levels of experience and skill. International Journal of Human-Computer Studies, 69, 155-169.

Poll, H. (Coord.) (2014). Pearson Student Mobile Device Survey 2014. National Report: College Students. Recuperado de http:/www.pearsoned.com/wp-content/uploads/ Pearson-HE-Student-Mobile-Device-Survey-PUBLIC-Report-051614.pdf

Sánchez Vera, Ma․, Prendes Espinosa, Maㅗ. P., \& Fernández Breis, J. (2013). Tecnologías semánticas para la evaluación en red: análisis de una experiencia con la herramienta OeLE. Revista de Investigación Educativa, 31(2), 447-464. Doi: http://dx.doi.org/10.6018/ rie.31.2.116721

Sevillano, M.르., \& Vázquez-Cano, E. (2015). The impact of digital mobile devices in Higher Education. Educational Technology \& Society, 18(1), 106-118.

UNESCO (2013). UNESCO Policy Guidelines for Mobile Learning. Paris: United Nations Educational, Scientific and Cultural Organization.

Vázquez-Cano, E. (2012). Mobile learning with Twitter to improve linguistic competence at secondary schools. The New educational Review, 29(3), 134-147.

Vázquez-Cano, E., Fombona, J., \& Fernández, A. (2013). Virtual Attendance: Analysis of an Audiovisual over IP System for Distance Learning in the Spanish Open University (UNED). The International Review of Research in Open and Distance Learning (IRRODL), 14(3), 402-426. 
Vázquez-Cano, E. (2014). Mobile Distance learning with Smartphones and Apps in Higher Education. Educational Sciences: Theory \& Practice, 14(4), 1-16. Doi: 10.12738/ est.2014.4.2012

Vázquez-Cano, E., \& Sevillano, M. (2014). Analysis of the didactic use of tablets in the European Higher Education Area. RUSC. Universities And Knowledge Society Journal, 11(3), 63-77.

Vladar, A., \& Fife, E. (2010). The growth of mobile social networking in the US. Intermedia, 38(3), 30-33.

Fecha de recepción: 05 de abril de 2015.

Fecha de revisión: 05 de abril de 2015.

Fecha de aceptación: 29 de marzo de 2016. 
